\title{
Chiroptical Hot Spots in Twisted Nanowire Plasmonic Oscillators
}

\section{Citation}

Tang, Yiqiao, Li Sun, and Adam Ezra Cohen. 2013. "Chiroptical Hot Spots in Twisted Nanowire Plasmonic Oscillators." Applied Physics Letters 102 (4): 043103.

\section{Published Version}

doi:10.1063/1.4789529

\section{Permanent link}

http://nrs.harvard.edu/urn-3:HUL.InstRepos:13454933

\section{Terms of Use}

This article was downloaded from Harvard University's DASH repository, and is made available under the terms and conditions applicable to Open Access Policy Articles, as set forth at http:// nrs.harvard.edu/urn-3:HUL.InstRepos:dash.current.terms-of-use\#OAP

\section{Share Your Story}

The Harvard community has made this article openly available.

Please share how this access benefits you. Submit a story.

\section{Accessibility}




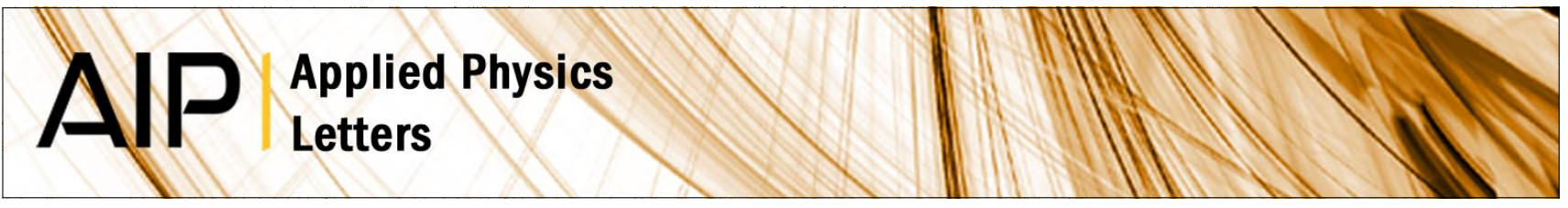

\section{Chiroptical hot spots in twisted nanowire plasmonic oscillators}

Yiqiao Tang, Li Sun, and Adam E. Cohen

Citation: Appl. Phys. Lett. 102, 043103 (2013); doi: 10.1063/1.4789529

View online: http://dx.doi.org/10.1063/1.4789529

View Table of Contents: http://apl.aip.org/resource/1/APPLAB/v102/i4

Published by the American Institute of Physics.

\section{Related Articles}

Fine-tuning of whispering gallery modes in on-chip silica microdisk resonators within a full spectral range Appl. Phys. Lett. 102, 041104 (2013)

The plasmonic J-pole antenna

Appl. Phys. Lett. 102, 033106 (2013)

Electro-optic sensor for specific absorption rate measurements

Appl. Phys. Lett. 102, 033502 (2013)

Cavity piezooptomechanics: Piezoelectrically excited, optically transduced optomechanical resonators Appl. Phys. Lett. 102, 021110 (2013)

Sub-diffraction optical coherent control of ultrafast electrical currents in antenna devices on GaAs Appl. Phys. Lett. 101, 251119 (2012)

\section{Additional information on Appl. Phys. Lett.}

Journal Homepage: http://apl.aip.org/

Journal Information: http://apl.aip.org/about/about_the_journal

Top downloads: http://apl.aip.org/features/most_downloaded

Information for Authors: http://apl.aip.org/authors

\section{ADVERTISEMENT}

\section{AIP Applied Physics Letters}

\section{EXPLORE WHAT'S NEW IN APL}

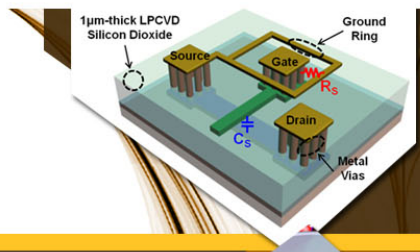

SURFACES AND INTERFACES

Focusing on physical, chemical, biological, structural, optical, magnetic and electrical properties of surfaces and interfaces, and more.. 


\title{
Chiroptical hot spots in twisted nanowire plasmonic oscillators
}

\author{
Yiqiao Tang, ${ }^{1}$ Li Sun, ${ }^{2}$ and Adam E. Cohen ${ }^{1,3, a)}$ \\ ${ }^{1}$ Department of Physics, Harvard University, Cambridge, Massachusetts 02138, USA \\ ${ }^{2}$ School of Engineering and Applied Science, Harvard University, Cambridge, Massachusetts 02138, USA \\ ${ }^{3}$ Department of Chemistry and Chemical Biology, Harvard University, Cambridge, Massachusetts 02138, USA
}

(Received 14 December 2012; accepted 11 January 2013; published online 28 January 2013)

\begin{abstract}
We image the chiroptical response of crossed nanowire junctions as a function of the vertical offset between the nanowires and the incident wavelength. These samples show chiroptical "hot spots" with strongly localized circular differential scattering at the nanowire crossing points. These chiroptical hot spots are not apparent in the spatially averaged spectra. The sign and magnitude of the chiroptical enhancement show a complex dependence on sample geometry, which we reproduce with a fully retarded analytical scattering model. These results suggest strategies for engineering devices with enhanced chiral light-matter interactions. (c) 2013 American Institute of Physics.

[http://dx.doi.org/10.1063/1.4789529]
\end{abstract}

The ability to control chiral light-matter interactions is critical for our understanding of fundamental physical symmetries ${ }^{1-3}$ and opens up new possibilities for applications in sensing, molecular structure determination, and optics, such as the design of circular polarizers ${ }^{4}$ and negative index metamaterials. ${ }^{5}$ In principle, metal nanostructures can bridge the mismatch between visible wavelengths and molecular dimensions by generating optical-frequency fields that vary over molecular-scale distances. ${ }^{6,7}$ Such fields can excite electric dipole-forbidden transitions in molecules, including those associated with chiroptical response.

Chiral metal nanostructures show strongly enhanced chiroptical properties, including strong circular differential scattering ${ }^{4,8-11}$ and asymmetric interactions with chiral molecules. $^{12,13}$ Spatially averaged measurements have yielded circular dichroism spectra that depended on the nanostructure geometry, but these measurements were not sensitive to spectral features that may be localized to particular regions of the sample. Metal nanostructures have long been known to generate dramatically enhanced local field intensities and gradients. ${ }^{14}$ We hypothesized that within regions of highly contorted fields one might find greatly enhanced chiroptical response, which was not seen in whole-field averages. We thus sought to create a chiral metal nanostructure where the chiroptical response could be probed as a function of space and device geometry.

In 1929, Kuhn introduced a simple classical model for a chiral molecule, consisting of two dipolar oscillators, displaced vertically from each other by a distance $h$, and rotated about the displacement axis by an angle $\theta$ (Figure 1), ${ }^{15}$ called the "twisted H" geometry. We created twisted $\mathrm{H}$ dipolar oscillators from crossed nanowires. We mapped the circular dichroism spectra as a function of $h$ and $\lambda$, and observed remarkable chiroptical "hot spots" at the crossing points of the nanowires.

To create devices with tunable spacing between the nanowires we used the technique of convex lens induced confinement (CLIC; Figure 2). ${ }^{16}$ We fabricated arrays of

\footnotetext{
${ }^{\text {a) }}$ Author to whom correspondence should be addressed. Electronic mail: cohen@chemistry.harvard.edu.
}

parallel metal nanowires on two fused silica substrates. The patterned substrates were aligned face to face with the nanowire axes oriented at $\pm 45^{\circ}$ relative to each other. An annular spacer with an inner radius of $10 \mathrm{~mm}$ created a $10 \mu \mathrm{m}$ air gap between the flat substrates. A convex lens pushed down on the top substrate, causing the substrate to bow downward until it made contact with the bottom substrate at a single point. Twisted $\mathrm{H}$ nanowire pairs were thus formed with vertical gaps that varied continuously from zero at the contact point to $10 \mu \mathrm{m}$ at the edge of the annulus. Due to the shallow curvature of the top substrate, translations of hundreds of microns in the plane of the sample led to changes in gap height of tens of nanometers.

The CLIC apparatus was mounted in a homemade microscope designed for spectroscopic polarimetry. A homemade Czerny-Turner monochromator provided illumination.

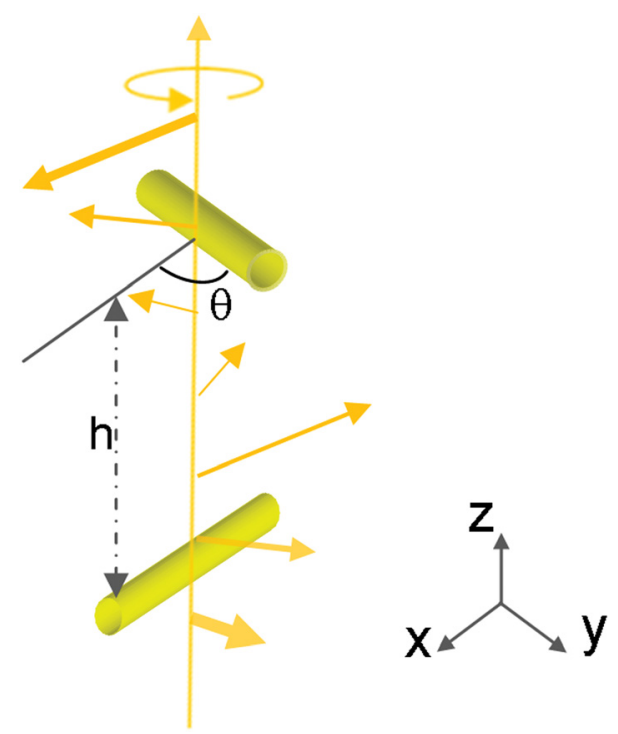

FIG. 1. Twisted $\mathrm{H}$ configuration of nanowire plasmonic oscillators. The nanowires were affixed to planar substrates in a sandwich geometry, and the top substrate was bowed down toward the bottom one to set the gap height $h$. In all experiments described here, $\theta= \pm 45^{\circ}$. The incident circularly polarized fields are shown in orange. 


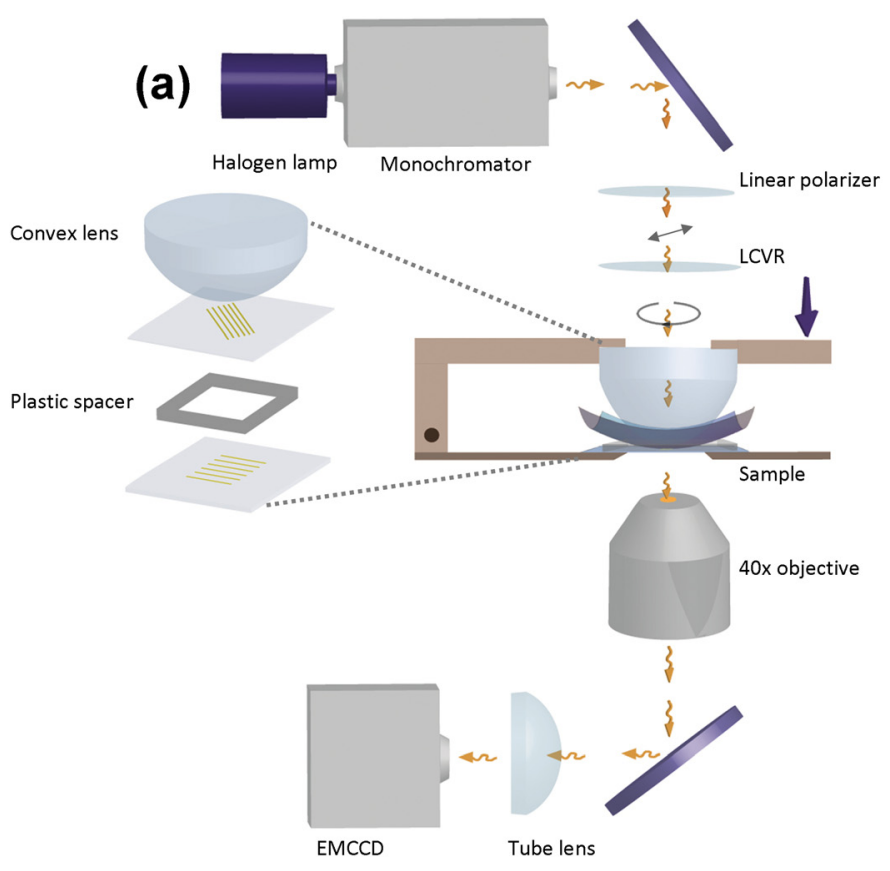

A liquid crystal variable retarder modulated the incident polarization in synchrony with video capture of transmittedlight images of the sample. For each data point, 40 images where acquired with alternating polarization. Signal from nanowire-free regions of the sample was used to correct for variations in illumination intensity. The microscope reported maps of the Jones matrices (linear and circular dichroism, linear and circular birefringence), with sub-micron spatial resolution and $<10 \mathrm{~nm}$ spectral resolution between wavelengths of 525 and $910 \mathrm{~nm}^{17}$

Figure 3(a) shows an image under transmitted monochromatic light $(630 \mathrm{~nm})$ of the crossed nanowire array. At low magnification $(4 \times$; inset) we observed a Newton's rings pattern due to interference of reflections from the top and bottom glass-air interfaces. At higher magnification $(40 \times)$, the crossed nanowire arrays were resolved as well. The square alignment marks enabled precise location of each high magnification image within the low magnification image. The gap height at every nanowire junction was then determined through reference to the Newton's rings pattern.

We acquired a series of images as in Figure 3(a), alternately under left- and right-circular polarized light (CPL). We then constructed a dissymmetry map, defined as

$$
g=\frac{I^{L}-I^{R}}{\frac{1}{2}\left(I^{L}+I^{R}\right)},
$$

where $I^{\mathrm{L}}$ and $I^{\mathrm{R}}$ represent the images under left- and rightCPL. To our surprise, "hot spots" appeared in this dissymmetry map at the nanowire crossings (Fig. 3(b)). The dissymmetry value at the hot spot varied with separation between the nanowires, and even changed sign at some gap heights (Figures 3(c) and 3(d)). Thus, the "handedness" of the twisted $\mathrm{H}$ did not uniquely determine the sign of the optical dissymmetry, even at a fixed wavelength. This tunability may be useful in nanophotonic devices where a simple linear motion of one component can change the qualitative behavior of the array. (b)

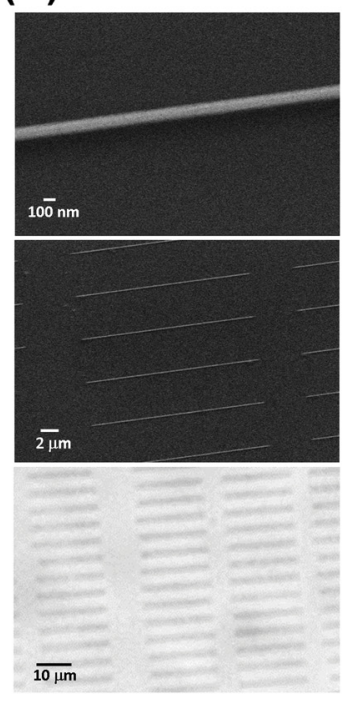

FIG. 2. Experimental setup. (a) CLIC system for creating a tunable plasmonic coupling. The sample consisted of two substrates containing linear arrays of nanowires. The substrates were arranged in a sandwich geometry, with the nanowires on the inside, and a thin annular spacer preventing contact. A mechanical vise pressed down on the top substrate via a convex lens, causing the two substrates to contact at a point. Linearly polarized monochromatic illumination was converted alternately to left- and right-circular polarization by a liquid crystal variable retarder (LCVR). Transmittedlight images of the sample were acquired on an electron-multiplying CCD (EMCCD) camera. (b) Nanowires used in the experiments. Top: high magnification SEM image; middle: lowmagnification SEM image; bottom: transmitted light image of a single nanowire array $(\lambda=630 \mathrm{~nm})$.
The dissymmetry map was dramatically different from the transmitted light images from which it was calculated. While the nanowires were clearly visible in the transmitted light images, only their crossings appeared in the dissymmetry map. High magnification views of the crossings (Figure 3(c)) showed a dissymmetry pattern consisting of concentric rings with dissymmetry of alternating sign. These features highlight the importance of obtaining spatially resolved maps of chiral metallic nanostructures. In a spatially averaged measurement, these features would be lost.

To explore further these chiral hot spots, we acquired circular dichroism images as a function of wavelength and gap height. We measured the dissymmetry factor at the nanowire crossing points, $g(h, \lambda)$ (Figure $4(\mathrm{a})$ ). This quantity oscillated as a function of $h$, with a period of $\lambda / 2$. Circular differential scattering is an interference effect, requiring a round-trip path of the light between the oscillators; hence the $\lambda / 2$ periodicity. However, the $h$-dependence was not sinusoidal, and furthermore the sign of $g$ depended on $h$ and $\lambda$ in a complex way. Switching the orientation of the top nanowires from $+45^{\circ}$ to $-45^{\circ}$ reversed the sign of $g(h, \lambda)$, as expected.

To interpret the surprising dependence of dissymmetry on gap height and wavelength, we next developed an analytical model of twisted $\mathrm{H}$ dipole oscillators, including fully retarded electrodynamic coupling, modeled on the work of Keller and Bustamante. ${ }^{18}$ We treated each rod as a pointlike anisotropic dipolar oscillator with frequency-dependent complex polarizability tensor

$$
\boldsymbol{\alpha}_{i}=\varepsilon_{0} A V R\left(\varphi_{i}\right)\left(\begin{array}{cc}
1 & 0 \\
0 & \rho e^{i \beta}
\end{array}\right) R\left(-\varphi_{i}\right)
$$

where $\rho$ and $\beta$ measure linear dichroism and linear birefringence, respectively, and the overall amplitude and phase of the dipole response are determined by a complex coefficient $A=A_{0} \mathrm{e}^{i \psi}$. These four parameters $\left(\rho, \beta, A_{0}\right.$, and $\left.\psi\right)$ depend on the frequency of illumination, the composition of the particle, and its shape. The rotation matrix $R\left(\phi_{i}\right)$ accounts for 
(a)

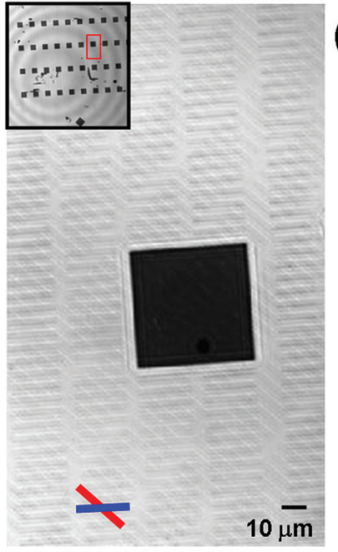

(b)

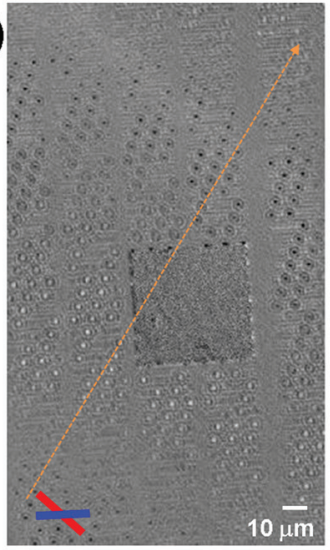

(c)

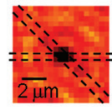

Gap

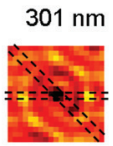

$556 \mathrm{~nm}$

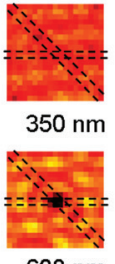

$608 \mathrm{~nm}$

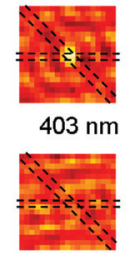

$659 \mathrm{~nm}$

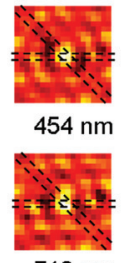

$710 \mathrm{~nm}$

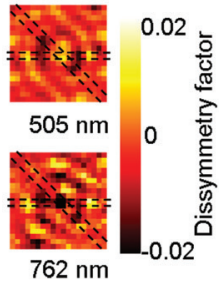

(d)

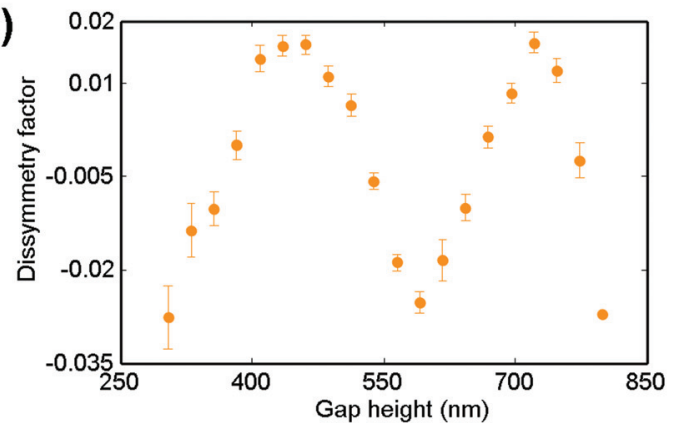

FIG. 3. Chiral hot-spots in twisted nanowire plasmonic oscillators. (a) Transmitted light image of a crossed nanowire array $(\lambda=630 \mathrm{~nm})$. Blue and red lines show the alignment of the nanowires. The black square in the middle is an alignment mark. Inset: low-magnification image showing circular Newton's rings interference pattern. This pattern was used to obtain a precise calibration of the gap at every nanowire crossing. (b) Dissymmetry map of the field of view shown in (a). Hot-spots were clearly visible at the junctions of the nanowires, while the nanowires themselves did not appear. The orange arrow points in the direction of increasing gap height. Within this field of view, the dissymmetry at the nanowire junctions changed sign. (c) Close-up images of the dissymmetry patterns at the nanowire junctions as a function of the gap height. Each image was constructed by averaging the dissymmetry maps of all junctions whose gaps were within $10 \mathrm{~nm}$ of the stated value. Dotted overlays show orientations of the nanowires. (d) Dissymmetry at the nanowire crossing as a function of gap height.

the orientation of the dipoles $\left(\phi_{1}=0, \phi_{2}= \pm 45^{\circ}\right) . V$ is an effective volume. We used our polarimetric microscope to measure these parameters as a function of wavelength on individual rods in a single layer. ${ }^{17}$

We then coupled the rods in the first Born approximation using the retarded electromagnetic Green's function as the field propagator. ${ }^{17}$ The fields scattered by the rods in the forward direction were added to the incident field to determine the intensity at the detector. Repeating this calculation for left- and right-CPL produced a prediction for the wavelengthand separation-dependent dissymmetry factor, Eq. (1).

Figure 4 shows measured and calculated dissymmetry factors at the nanowire junctions as a function of gap height and wavelength. The theoretical fitting and data coincide (a)

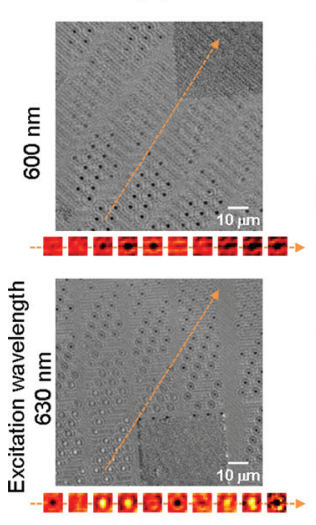

(b)

(c)
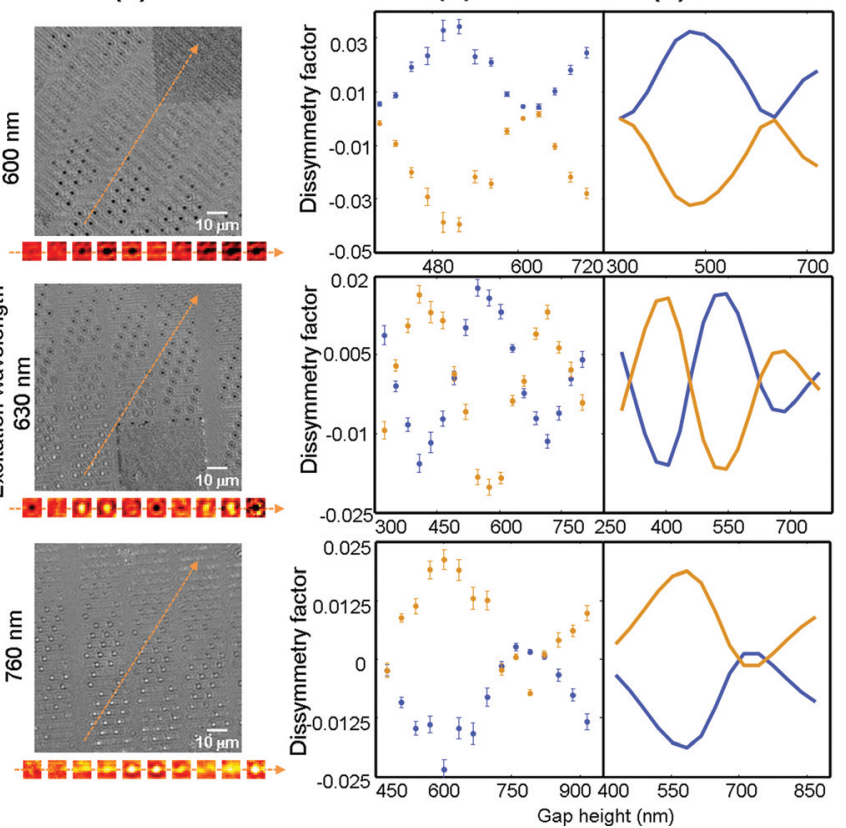

FIG. 4. Comparison of measured dissymmetry factors to theory. (a) Dissymmetry maps were measured at three wavelengths $(600,630,760 \mathrm{~nm})$, all for the same chiral configuration. Maps of the dissymmetry factor were calculated at nanowire junctions, as a function of separation (bottom). (b) Dissymmetry factor as a function of gap height. (c) Theoretical predictions of dissymmetry based on a retarded coupled oscillator model.

closely for both enantiomeric configurations $\left(\theta= \pm 45^{\circ}\right)$. Table $\mathrm{S} 1$ gives fitting parameters. Thus, the optical dissymmetry of twisted nanowire plasmonic oscillators is well described by retarded dipolar interactions in the first Born approximation.

At gaps smaller than those studied here, our analytical approach may need extension in two ways. First, higherorder couplings of the rods may become important, i.e., one should solve for the fields self-consistently rather than in the single-scattering limit. Second, the point-dipole approximation may need extension to include higher order Floquet modes in the nanowires.

A key challenge in designing chiral plasmonic sensors has been the difficulty of placing molecules in the region of strongest chiral field; often these regions reside in the dielectric support. The sandwich geometry of the CLIC device enables one to flow into the gap any test molecule of interest, and to disassemble and clean the nanowires between uses. We speculate that chiral molecules may show strongly enhanced circular dichroism when probed with the highly twisted electromagnetic fields that arise in the twisted nanojunctions. The CLIC geometry enables one to probe such phenomena as a function of gap height, without the need to fabricate new devices for each height.

In metamaterial research, the conventional fabrication processes create rigid structures with fixed frequency responses. Greater flexibility and functionality, as required for many practical uses, may be achieved by controlling the effective electromagnetic parameters of the artificial medium through externally tunable components. One approach is through integration of the dielectric/metallic structures with other classes of materials such as liquid crystals, with tunable dielectric properties. ${ }^{19}$ Another approach, "structural tuning," is based on a continuous adjustment of the lattice 
structure. For example, the optical properties of metal nanostructures on a compliant polydimethylsiloxane (PDMS) substrate could be tuned by stretching the PDMS. ${ }^{20}$ Chiral structures are by necessity three dimensional, and the experiments presented here demonstrate mechanical tuning in a three-dimensional plasmonic structure. The continuously variable gap of the CLIC geometry may prove useful in other experiments where one wishes to probe the optical properties of nanostructures as a function of their separation.

The appearance of chiroptical hot-spots in a chiral nanowire array underscores the importance of real-space imaging in characterizing plasmonic nanostructures and points toward the possibility of enhanced chiral sensing using crossed nanowire plasmonic structures.

We thank Jonathan Fan and Federico Capasso for helpful discussions, and Nan Niu for help with the nanofabrication. This work was supported by DARPA YFA Grant No. N66001-091-2104 and ONR YIP Grant No. N000140910868. Sample preparation was performed at the Harvard Faculty of Arts and Sciences (FAS) Center for Nanoscale Systems, a member of the National Nanotechnology Infrastructure Network, supported by NSF Award No. ECS-0335765.

${ }^{1}$ Y. Tang and A. E. Cohen, Phys. Rev. Lett. 104, 163901 (2010).

${ }^{2}$ A. S. Schwanecke, A. Krasavin, D. M. Bagnall, A. Potts, A. V. Zayats, and N. I. Zheludev, Phys. Rev. Lett. 91, 247404 (2003).
${ }^{3}$ K. Y. Bliokh and F. Nori, Phys. Rev. A 83, 021803 (2011).

${ }^{4}$ J. K. Gansel, M. Thiel, M. S. Rill, M. Decker, K. Bade, V. Saile, G. Von Freymann, S. Linden, and M. Wegener, Science 325, 1513 (2009).

${ }^{5}$ S. Zhang, Y. S. Park, J. Li, X. Lu, W. Zhang, and X. Zhang, Phys. Rev. Lett. 102, 23901 (2009).

${ }^{6}$ N. Yang, Y. Tang, and A. E. Cohen, Nano Today 4, 269 (2009).

${ }^{7}$ M. Aeschlimann, M. Bauer, D. Bayer, T. Brixner, F. J. Garcia de Abajo, W. Pfeiffer, M. Rohmer, C. Spindler, and F. Steeb, Nature 446, 301 (2007).

${ }^{8}$ B. M. Maoz, A. Ben Moshe, D. Vestler, O. Bar-Elli, and G. Markovich, Nano Lett. 12, 2357 (2012).

${ }^{9}$ A. O. Govorov, Y. K. Gun'ko, J. M. Slocik, V. A. Gérard, Z. Fan, and R. R. Naik, J. Mater.Chem. 21, 16806 (2011).

${ }^{10}$ M. Hentschel, M. Schäferling, T. Weiss, H. G. Kuball, N. Liu, and H. W. Giessen, Nano Lett. 12, 2542 (2012).

${ }^{11}$ Y. Zhao, M. A. Belkin, and A. Alù, Nature Commun. 3, 870 (2012).

${ }^{12}$ E. Hendry, T. Carpy, J. Johnston, M. Popland, R. V. Mikhaylovskiy, A. J. Lapthorn, S. M. Kelly, L. D. Barron, N. Gadegaard, and M. Kadodwala, Nat. Nanotech. 5, 783 (2010).

${ }^{13}$ N. Abdulrahman, Z. Fan, T. Tonooka, S. Kelly, N. Gadegaard, E. Hendry, A. O. Govorov, and M. Kadodwala, Nano Lett. 12, 977 (2012).

${ }^{14}$ P. K. Jain, D. Ghosh, R. Baer, E. Rabani, and A. P. Alivisatos, Proc. Natl. Acad. Sci. 109, 8016 (2012).

${ }^{15}$ W. Kuhn, Z. Phys. Chem. B 3-4, 15 (1929).

${ }^{16}$ S. R. Leslie, A. P. Fields, and A. E. Cohen, Anal. Chem. 82, 6224 (2010).

${ }^{17}$ See supplementary material at http://dx.doi.org/10.1063/1.4789529 for fabrication methods, data analysis, and analytical theory.

${ }^{18}$ D. Keller and C. Bustamante, J. Chem. Phys. 84, 2961 (1986).

${ }^{19}$ W. Dickson, G. A. Wurtz, P. R. Evans, R. J. Pollard, and A. V. Zayats, Nano Lett. 8, 281 (2008).

${ }^{20}$ I. M. Pryce, K. Aydin, Y. A. Kelaita, R. M. Briggs, and H. A. Atwater, Nano Lett. 10, 4222 (2010). 